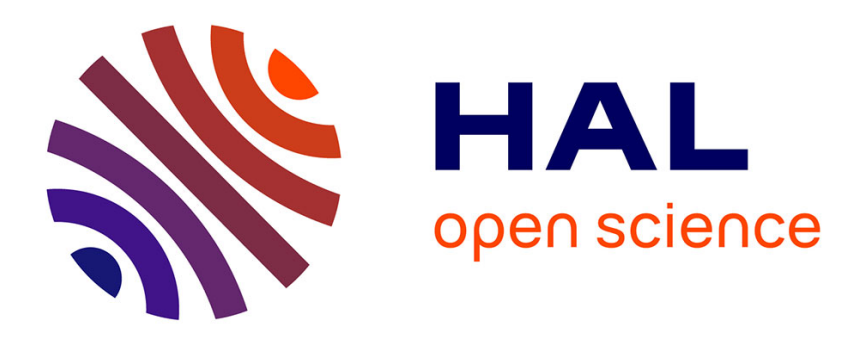

\title{
The Switching Dynamics of the Plasma Addressed Liquid Crystal Display
}

\author{
K. Ilcisin, T. Buzak, G. Parker
}

\section{To cite this version:}

K. Ilcisin, T. Buzak, G. Parker. The Switching Dynamics of the Plasma Addressed Liquid Crystal Display. Journal de Physique IV Proceedings, 1997, 07 (C4), pp.C5-225-C5-233. 10.1051/jp4:1997418 . jpa-00255574

\section{HAL Id: jpa-00255574 https://hal.science/jpa-00255574}

Submitted on 1 Jan 1997

HAL is a multi-disciplinary open access archive for the deposit and dissemination of scientific research documents, whether they are published or not. The documents may come from teaching and research institutions in France or abroad, or from public or private research centers.
L'archive ouverte pluridisciplinaire HAL, est destinée au dépôt et à la diffusion de documents scientifiques de niveau recherche, publiés ou non, émanant des établissements d'enseignement et de recherche français ou étrangers, des laboratoires publics ou privés. 


\title{
The Switching Dynamics of the Plasma Addressed Liquid Crystal Display
}

\author{
K.J. Ilcisin, T.S. Buzak and G.J. Parker*
}

Technical Visions Inc., M/S 46-944, 140010 S.W. Karl Braun Dr., Beaverton, OR 97077, U.S.A.

* Lawrence Livermore National Laboratory, P.O. Box 808, L-418, Livermore, CA 94551, U.S.A.

\begin{abstract}
The plasma addressed liquid crystal display (PLAC) is a flat, large area, full-colour television and monitor technology made possible through the unique application of the properties of an ionized gas. Liquid crystal displays require an active switching element at the location of each pixel. The PALC display uses a plasma switch to address each of the display pixels, replacing typically used semiconductor devices. The use of the plasma switch permits the manufacture, in very large sizes, of a display possessing excellent image quality. The performance of the PALC display is intrinsically related to the behaviour of the plasma used as the switching element. This paper reviews the trends that led to the development of the PALC display, describes the operating principals, and examines, in detail, one of the many areas of ongoing plasma physics research. The successful development of a model, which agrees with the measured decay behaviour of the plasma, contributed to a significant improvement in the performance of the PALC display.
\end{abstract}

\section{INTRODUCTION}

For decades, "Hang on the wall" TVs have been conjured up in the minds of display researchers and science fiction writers alike. The writers have recently been infuriated by the limits being placed on their imaginations as display researchers have been successful in developing flat panel displays (FPD). Several display technologies have been invented by groups intent on replacing the CRT. These devices are found in a multitude of applications from calculators to lap-top computer monitors. Most of these technologies, however, are limited in maximum diagonal size to less than $65 \mathrm{~cm}$ and are not suitable for use as TVs or desktop monitors. Even the ubiquitous Thin Film Transistor (TFT) active matrix liquid crystal display (AMLCD) - the performance leader in FPDs used in the majority of the world's lap-top computersbecomes exceedingly difficult to manufacture in sizes approaching $50 \mathrm{~cm}$. This size limitation rises from the use of semiconductor devices and their related manufacturing constraints. Wafer scale integration on substrates with surface areas exceeding $1,000 \mathrm{~cm}^{2}$ is a tremendously difficult task.

Two alternative display technologies have emerged as the replacement for the CRT in large sizes. Both of these technologies have in common their use of a plasma, and are referred to as plasma addressed displays. The first is the plasma addressed liquid crystal display or PALC, the other is the plasma addressed phosphor display or PDP. The difference between these two technologies is the reliance on different plasma phenomena for their operation. PDP panels utilize the UV emission from a plasma to excite phosphor[1]. In contrast, the PALC display uses the electrical properties of a glow discharge to act as a high performance, and easily manufactured, switching device[2]. PALC technology, invented in 1990, permits the manufacture of a very large AMLCDs The PALC display embodies the performance characteristics of current AMLCDs-high contrast, high brightness, full motion video and full gray scale fidelity-but without the size limitation. As a testament to the ease of scalability for the PALC display technology, it is edifying to compare the recent advances in the size of the PALC display. In 1990 a $15 \mathrm{~cm}$ display was introduced, a $40 \mathrm{~cm}$ PALC monitor was demonstrated in 1993[3], a $64 \mathrm{~cm}$ wide format TV was developed in 1996[4], and most recently, plans were announced for the demonstration of an over 100 $\mathrm{cm}$ PALC display in early 1998. For comparison, size increases for semiconductor devices have averaged about $2 \mathrm{~cm}$ a year during the past two decades.

Article published online by EDP Sciences and available at http://dx.doi.org/10.1051/jp4:1997418 
We organized this paper as follows. In Section 2, we detail the operating principles of a PALC display. In Section 3, we describe the computational model used to investigate the dynamics of the plasma switch. Finally, in Section 4, we illustrate how the knowledge gained from the computations and measurements resulted in a significant improvement in the performance of the PALC display.

\section{PLASMA ADDRESSING OF LIQUID CRYSTAL DISPLAYS}

The quality of the visual image of an AMLCD is influenced by the performance and properties of the active matrix switch. For TFT AMLCDs, which rely on semiconductor switches, the physics of the device is reasonably well understood. In contrast, the switch in the PALC display represents a relatively new regime of study, as the physical geometries, time scales of interest, and operating conditions differ significantly from typical experimental devices. The following sections illustrate how a glow discharge functions as an active matrix switch and how the properties of this switch influence display performance.

\subsection{The Glow Discharge Tube as a Three Terminal Switch}

The plasma addressing technique relies on the properties of a confined, ionized gas and is embodied in a structure that can be used as an electrical switch. It is well known, from probe measurements, that the plasma potential of a cylindrically symmetric discharge tube is very near the anode potential for most of the tube volume, except near the cathode region where one measures the profile of the cathode fall. This effect, allows one to construct a hypothetical three terminal switch using a discharge tube and a voltage probe as illustrated in Figure 1.

The cathode connection can be thought of as a control or "gate" electrode. When the cathode voltage is switched on and the gas is ionized, the probe electrode appears electrically connected to the anode, here shown at ground potential. This would permit charging of the sampling capacitor. When the control electrode voltage is reduced below the plasma sustaining voltage, the plasma is extinguished and the probe electrode is no longer connected to the grounded anode. The charge stored on the sampling capacitor would remain fixed. The device sketched in Figure 1 behaves like a simple sample-and-hold circuit.

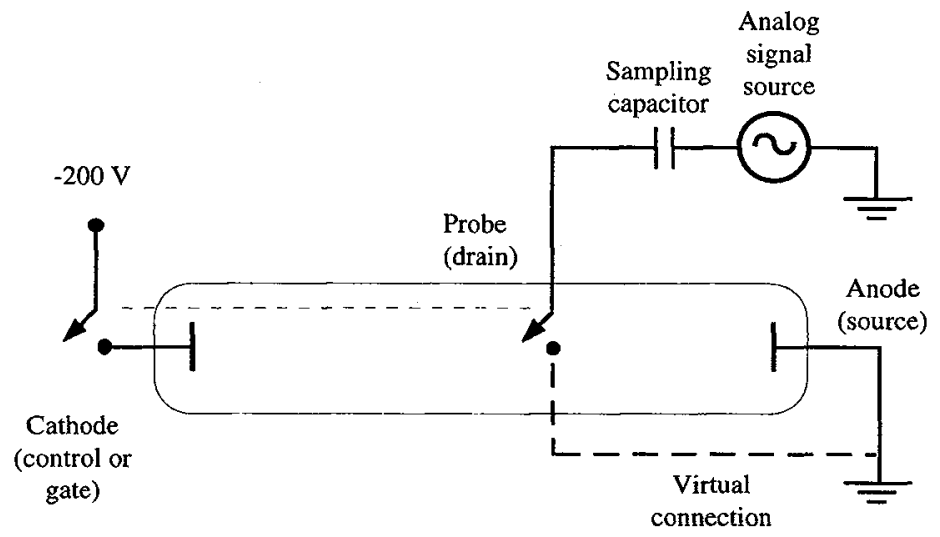

Figure 1: A sketch of a discharge tube configured as a sample-and-hold circuit using the tube as a three terminal.switch.

The circuit would work equally well if the plasma switch were replaced by a conventional silicon fieldeffect transistor (FET). When compared to a FET switch, the plasma cathode electrode plays a role similar to a transistor gate electrode; the anode and probe electrodes take on roles similar to the transistor source and drain electrodes; and the plasma sustain voltage is analogous to the gate-source threshold voltage. 


\subsection{Plasma Addressing of a Liquid Crystal Display}

A description of how a display device can be developed utilizing the switching device described in the previous section can be accomplished with the aid of Figure 2 which illustrates a partial cross-section of a PALC display. An electro-optic layer-a twisted nematic liquid crystal layer-and a protective dielectric layer are contained between two sheets of glass. The top sheet is patterned with transparent indium-tinoxide (ITO) conductive stripes; the bottom sheet has in its surface parallel grooves or channels. Each channel contains two parallel electrodes that run the entire channel length. The anodes are shown as grounded. The cathodes are partially hidden by the channel walls and electrically connected to the strobe drivers. The function of the "probe connection" is achieved by the storage of charge on the bottom surface of the dielectric which acts as the "bottom" of the pixel capacitor.

Active matrix displays update images by writing each horizontal line of an image frame sequentially. During one horizontal line time, analog data corresponding to a single line of video appears on the data outputs and the transparent conductors. Except for the one row where the gas is ionized, the analog voltage has no effect on light transmissivity of the liquid crystal since there is no electrical connection or conductor on the bottom side of the liquid crystal. Along the line above the ionized channel, however, the pixel capacitance can charge to the value determined by the data voltage. In effect, the plasma completes the electrical circuit between the data electrodes and the grounded channel anode.

For each pixel along a channel, the gas discharge functions as an electrical switch that changes between a conducting (plasma) state and a nonconducting (de-ionized) state in response to the applied data strobe signal. Analog data values are sampled and held in a row-by-row basis, sequentially addressing an entire image field of the liquid crystal layer. It is important to note that this is not an emissive plasma display: there is no image in the barely visible plasma glow. Power consumption is low: only one line of plasma is on at any given time, and then on only for a fraction of a horizontal line time.

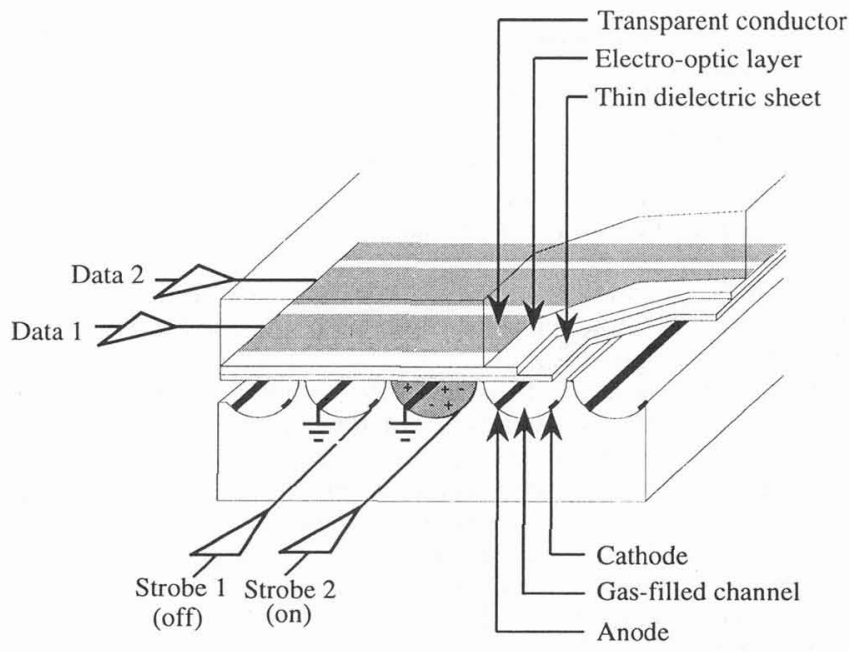

Figure 2: $\quad$ A partial cross-section of a PALC panel showing several channels and data electrodes. Channel 2 has a strobe voltage applied, filling that channel with conductive ions and electrons. Voltages from the data electrodes are captured at the intersections where the data electrodes cross channel 2.

\subsection{Addressability, Data Capture, and Switching Speed Requirements}

The addressability of a display - the number of lines that can be addressed in a single frame time-depends upon the speed with which any single line can be addressed. A modern high information content display, like a computer display or a high definition television display, requires an addressability of at least 1,000 lines with $60 \mathrm{~Hz}$ frame rates. This implies a minimum line time of approximately $16.6 \mu \mathrm{s}$ for progressively scanned displays, and $33.3 \mu$ s for interlaced displays. 
For a PALC display, line time is determined by the sum of the plasma initiation, data capture, and plasma decay times. Figure 3 illustrates this relationship.

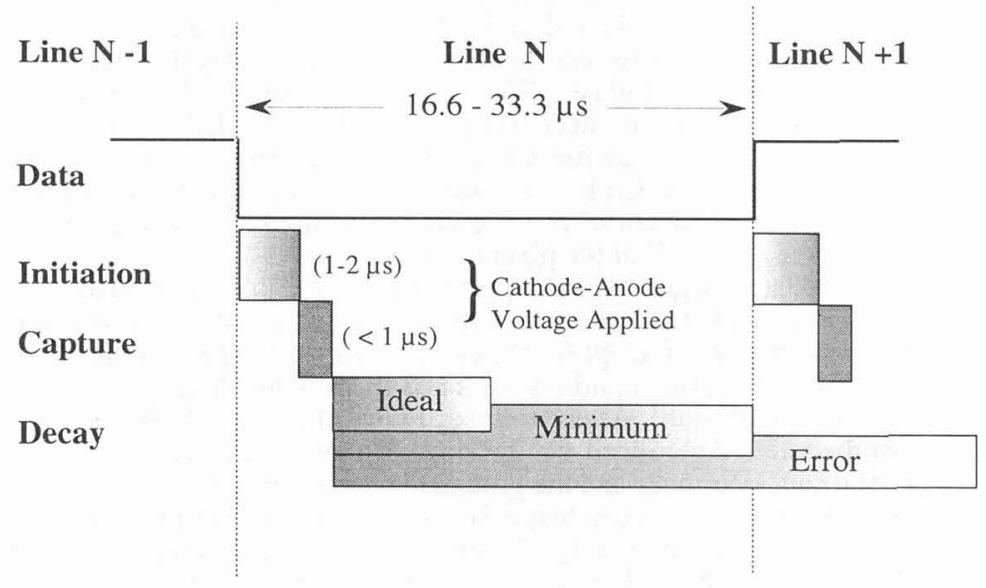

Figure 3: Horizontal line time requirements. One horizontal line time contains time segments for plasma initiation, data capture, and plasma decay.

The initiation time is the time it takes to turn the plasma on. This time is typically one to two microseconds. Although the initiation time can be established before the beginning of line time, the savings of time is minimal given the short time required for initiation.

The data capture time is the time for plasma to reach a steady state condition which is referred to as the active phase of the discharge. During this time, the pixel capacitance is charged to the value nominally close to that of the data voltage. Because of the low value of the pixel capacitance, data capture is very fast and can usually be held to less than one microsecond.

The most important time segment in a line time is the plasma decay time. The plasma must decay to a high resistivity before the image data can be changed to the values required for the next line. If the data is changed before the plasma has had an opportunity to decay, data from the subsequent line will be incorrectly captured. This is shown schematically in Figure 4, where for simplicity, the sheath layer present at the end of the active discharge was ignored.

Figure 4a) shows the conditions that exist just after the cathode voltage has been removed. A large density of charged particles exists in the gas volume, but there is nominally no net charge except at the surfaces. Correspondingly, the electric field exists predominately across the LC and dielectric layers and terminates near the bottom surface of the dielectric where a net negative surface charge layer is present for an applied positive data voltage. The voltage across the LC and dielectric layers is much larger than the applied voltage $V_{1}$ at $t=0$. This is due to the fact that the initial charge distribution reflects the cathode potential during the active phase. If the data voltage remains constant, then all of the free charge will diffuse to a surface or recombine. If no changes to the data voltage are made, the situation at $t=\infty$ is shown in Figure $4 \mathrm{~b})$. A surface charge layer at the bottom of the dielectric surface produces an electric field across the LC layer corresponding to the applied data voltage $V_{1}$. The gas volume is essentially field free.

In Figure $4 c$ ), we examine the situation where at an intermediate time $T_{h}$ the data voltage $V_{1}$ is switched to a smaller value $V_{2}$. At $T_{h}$ the surface charge layer is similar to that $t=\infty$, and an electric field is impressed in the discharge volume. Although the net charge density is nominally zero, there can still be a large charged particle density. These charges are created through processes such as metastable-metastable collisions in the afterglow and will move in response to this applied field. In Figure 4d) we see that a change in applied voltage results in a different charge density and electric field at $t=\infty$. However, the final surface charge density, field and potential across the LC and dielectric layers is not equal to $\mathrm{V}_{2}$ but to some admixture of $V_{1}$ and $V_{2}$. Thus, if the plasma has not completely decayed before the data voltage is changed for the subsequent horizontal line, an error occurs in the stored charge. Under these conditions, incorrect data capture can only be avoided by extending the line time, resulting in lower addressability. 


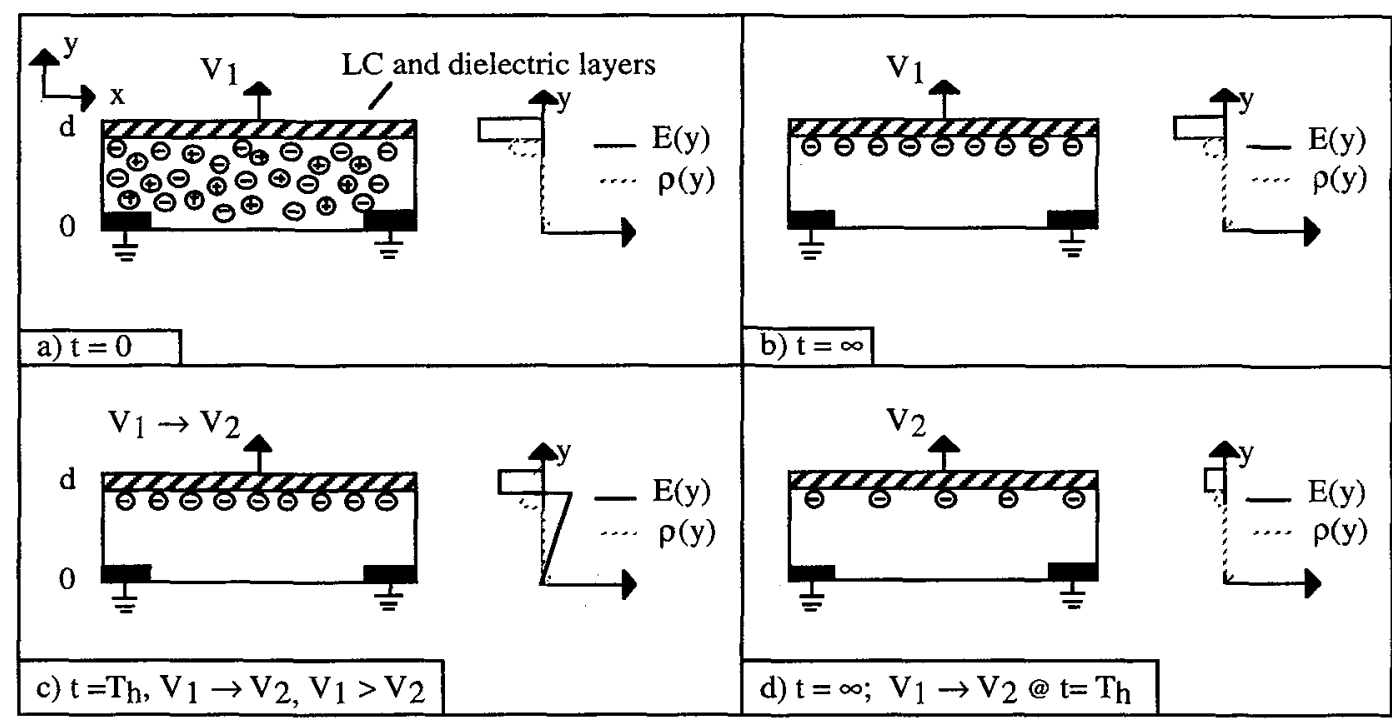

Figure 4: Figures a) and b) sketch the evolution of the charge and field profiles when the applied data voltage remains constant during the plasma decay period. Figures $\mathrm{c}$ ) and d) illustrate the changes that occur when the data voltage is switched during the decay phase.

\section{PLASMA COMPUTATIONAL MODEL}

Due to the size and structure of the plasma channel in a typical PALC display, many experimental measurements are difficult, if not impossible. Accordingly, an efficient computational model of the PALC display was developed to further the fundamental understanding of the device characteristics and possible approaches to improving the performance. In this section, we first outline the computational method used to simulate the plasma and then describe some of the results from the model.

\subsection{Computational Model}

The computational approach used in simulation of the plasma channel is a formidable task. During the 'active' or 'on' state of the plasma channel, large variations of the electric field occur inside a small plasma region. The typical approach of using hydrodynamic equations with the local field approximation (where the electron collision rates depend only on the ratio of the electric field and neutral number density) would lead to large errors in collision rates spatially, temporally, and in magnitude. In addition, during the 'afterglow' or 'off' state of the plasma channel high energy electrons are produced from metastablemetastable atom ionization thus making the electron energy distribution function non-Maxwellian which is what is usually assumed in such schemes. In contrast, a completely kinetic approach where the Boltzmann equation is solved for each particle species would be completely computationally unfeasible at the large neutral densities due to the extreme range in time scales: electron collision $\left(\sim 10^{-12} \mathrm{~s}\right)$ to neutral diffusion $\left(\sim 10^{-4} \mathrm{~s}\right)$ times. To overcome these computational obstacles, a hybrid approach was used. In this scheme, the hydrodynamic equations along with Poisson's equation are solved semi-implicitly. Instead of assuming an electron energy distribution function, which is needed to close the system of equations, it is calculated periodically according to the instantaneous field and particle density profiles. Once computed, various moments are computed and used to complete the hydrodynamic transport equations.

The model used to solve the hydrodynamic equations along with Poisson's equation has been discussed elsewhere[6] and so we only outline the basic equations here. For each charge species, the continuity equation is solved: 


$$
\frac{\partial n}{\partial t}=-\vec{\nabla} \cdot n \vec{V}+R
$$

where $n$ is the species number density, $\vec{V}$ is the drift velocity and $R$ is the net production rate per unit volume. Neutral species are assumed to be described by the diffusion equation:

$$
\frac{\partial n}{\partial t}-\nabla \mathrm{D} \cdot \nabla n=R
$$

where $\mathrm{D}$ is the diffusion coefficient. The electron drift velocity is assumed to be described by the drift/diffusion approximation, while the ion drift velocities are solved via solution of the momentum equation:

$$
\frac{\partial n \vec{V}}{\partial t}=-\vec{\nabla} \cdot(n \vec{v} \vec{v})+\frac{n q \vec{E}}{\mathrm{~m}}-\frac{1}{\mathrm{~m}} \vec{\nabla} n k T_{i}-\sum_{j} n \vec{v} v_{j}
$$

where $\mathrm{q}(\mathrm{m})$ is the charge (mass) of the ion, $\mathrm{E}$ is the electric field, $v_{j}$ is the collision frequency of $\mathrm{j}^{\prime}$ th ionneutral collision and $T_{i}$ is the ion temperature, which is assumed to be constant and uniform. Due to fierce ion-neutral collisions, this approximation is valid and therefore the ion energy equation does not need to be solved. In addition, the electron energy equation is redundant since we will be calculating the actual electron energy distribution function directly.

Ion and metastable impact onto the electrodes and dielectric surfaces can result in electron emission and this is simply characterized by a number, $\gamma$, which is the probability that an electron will be emitted for each ion and/or metastable impinging on a surface element. Obviously, different surfaces and species combinations have different values of gamma.

Finally, Poisson's equation,

$$
\vec{\nabla} \cdot \varepsilon \vec{\nabla} \Phi=-\vec{\nabla} \cdot \varepsilon \overrightarrow{\mathrm{E}}=\sum_{k} q_{k} n_{k}
$$

where $\Phi$ is the electrostatic potential and $\varepsilon$ is the permitivity of free space, is solved subject to the boundary conditions. To avoid tight numerical constraints on the time step, Poisson's equation along with the electron continuity equations are solved simultaneously[6]. As mentioned previously, the electron energy distribution function (EEDF) is calculated periodically according to the instantaneous electric field and particle density profile. From this EEDF, the electron collision frequencies and temperature profiles are computed and used to advance the hydrodynamic equations until the fields and/or particle density profiles change significantly so that a new EEDF is needed. The method chosen to calculate the EEDF is by use of a Monte Carlo[7] technique which uses a fourth order Runge-Kutta integrator[8] for advancing the electron trajectory and uses a null-collision technique[7,9] for collisions. However, to increase the computational efficiency of the Monte Carlo technique, electrons are split into two groups. The first group is unconfined electrons which are defined to be electrons whose total energy (kinetic plus potential) is greater than the minimum confining potential. The second group consists of all other electrons. If there is no confining potential, then the number of electrons in the second group is zero. Unconfined electrons, by definition, have a finite lifetime in the plasma region-either they escape to a surface or they lose sufficient kinetic energy to become confined. Unconfined electrons are initially produced from electrons emitted off surfaces from ion and/or metastable impact or from heavy particle collisions in the plasma bulk which lead to ionization. A large number of unconfined electrons $(-1000 \mathrm{~s})$ are launched according to the emission and heavy particle ionization collision profiles. The EEDF and total time are tracked until they either leave the discharge or become confined electrons. Any electrons produced in electron-impact ionization are also followed. A smaller number $(\sim 100 \mathrm{~s})$ of confined electrons are then followed which are initially launched according to the newly confined electron profile and the previously saved confined EEDF from the last iteration. These electrons are followed, tracking their EEDF and total time, until either they leave the discharge (either by having a superelastic or Coulomb collision which transfers the needed kinetic energy), become attached, or the total time followed reaches the time between calls to the Monte Carlo routine. The computational advantage is that the confined electrons are low energy electrons which do not participate in the plasma chemistry and are expensive because they must be followed for a long time. Following only a few of the confined electrons thus represents a large savings in computational time. After following each group of electrons, we have determined two EEDFs: confined and unconfined electrons. However, we scale the confined EEDF before adding it together with the unconfined EEDF to get the full EEDF. This combined function is then used to compute the electron temperature and collision frequency profiles. This scaling is found by using the hydrodynamic electron density. From the instantaneous electron number density profile, the total number of electrons in the discharge is computed by a simple volume integral. This number must be equal to the number of unconfined electrons plus the number of confined electrons. The number of unconfined electrons is simply the rate (number/s) they are being created (surface emission plus 
volume heavy particle ionization collisions) times the average lifetime of these electrons (and their progeny) in the discharge. The difference between these two numbers must be the number of confined electrons and is how the scaling of the confined EEDF is found.

\subsection{Results of Computational Model}
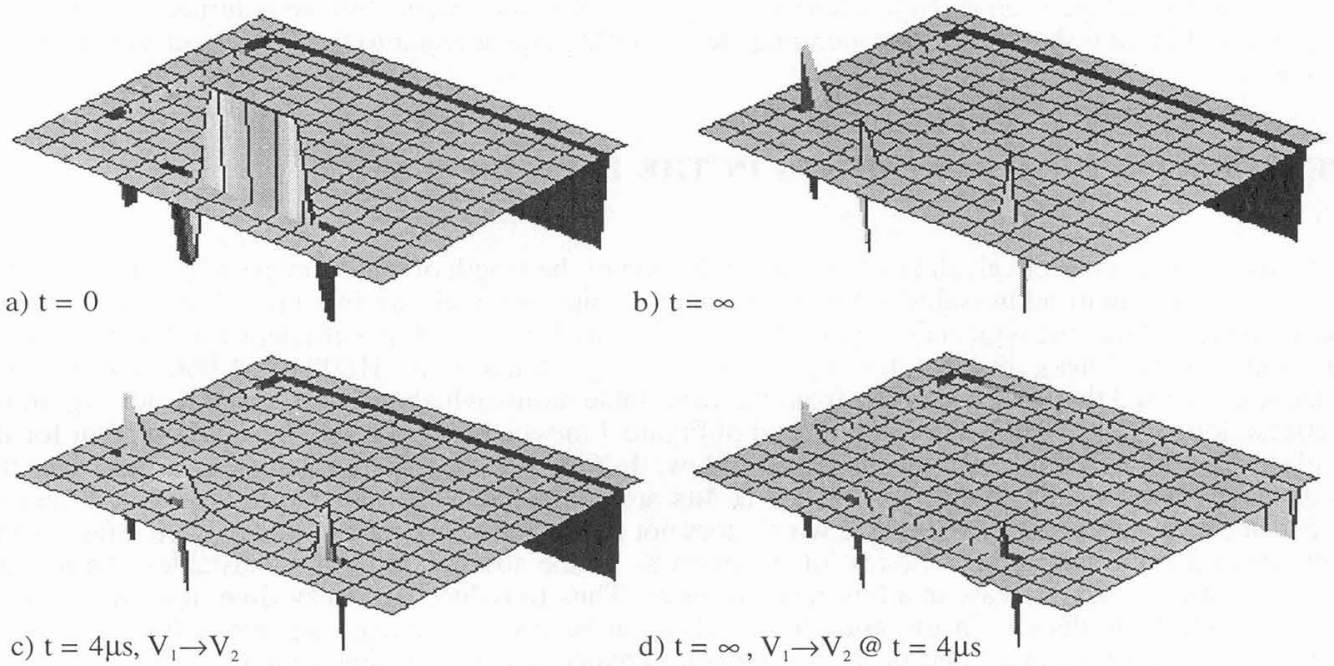

d) $\mathrm{t}=\infty, \mathrm{V}_{1} \rightarrow \mathrm{V}_{2} @ \mathrm{t}=4 \mu \mathrm{s}$

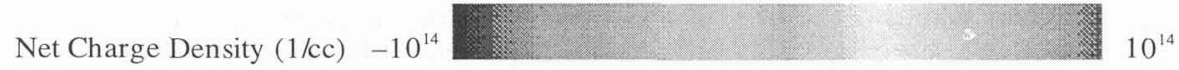

Figure 5: Figures $5 a$ ) and $b$ ) illustrate the charge density at $t=0$ and $t=\infty$ for a fixed data voltage.. Figures 5 c) and d) show the profiles at $t=4 \mu \mathrm{s}$ when the data voltage changes from $V_{1}$ to $V_{2}$ and the resultant profiles at $t=\infty$.

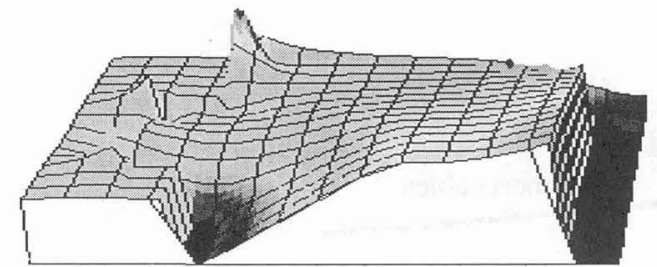

a) $t=0$

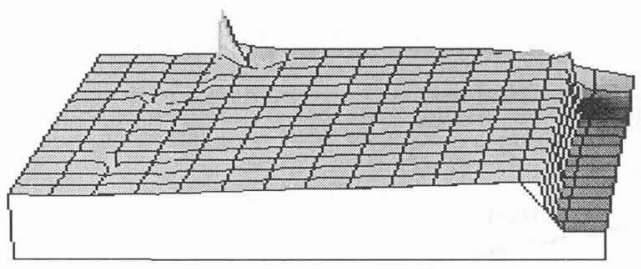

c) $\mathrm{t}=4 \mu \mathrm{s}, \mathrm{V}_{1} \rightarrow \mathrm{V}_{2}$

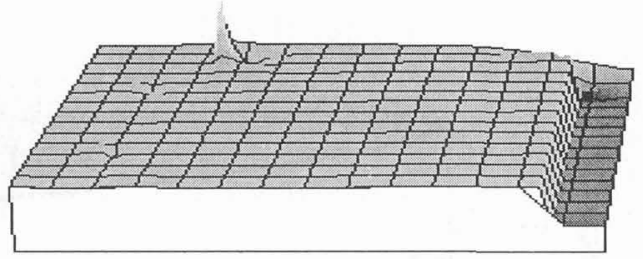

b) $t=\infty$

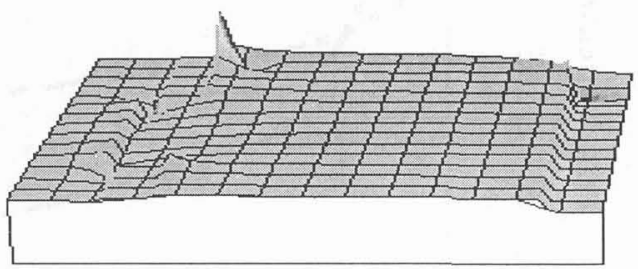

d) $\mathrm{t}=\infty, \mathrm{V}_{1} \rightarrow \mathrm{V}_{2} @ \mathrm{t}=4 \mu \mathrm{s}$

Electric Field (V/um)

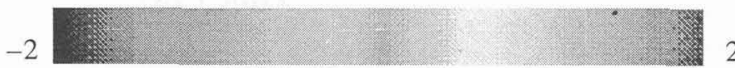

Figure 6: Figures 6 a) and b) illustrate the electric field profile at $t=0$ and $t=\infty$ for a fixed data voltage.. Figures $6 \mathrm{c}$ ) and d) show the profiles at $t=4 \mu$ s when the data voltage changes from $V_{1}$ to $V_{2}$ and the resultant profiles at $t=\infty$. 
In this section we present some of the results of the computational model which was presented above. The model was used for both the 'active' and 'afterglow' discharge. Results from the afterglow period are presented here. The simulation starts by specifying the voltage on the electrodes and the voltage on the address electrode. Once steady state solution is achieved corresponding to the end of the data capture phase in Figure 3, the applied sustaining voltage across the electrodes is removed and the plasma is allowed to decay. Figure 5 shows the charge density when the sustain voltage is first turned off $(t=0)$ and the final profile at long time $(t=\infty)$ when the address voltage is kept constant. Also shown is the charge density when the address voltage is changed to a lower voltage $4 \mu$ s after the sustain voltage is turned off and again at long times. Figure 6 shows the corresponding electric field perpendicular to the address electrode for the same cases.

\section{PERFORMANCE IMPROVEMENTS IN THE PALC DISPLAY}

The results of these types of calculations give an indication of the length of the decay period and are used to determine the maximum addressability for a particular architecture and gas mixture. Measurements and modeling indicated that the typical decay time for pure He was $20 \mu$ s which is sufficient for NTSC or VGA addressability (480 lines), but not for high addressability monitors or HDTV ( $>1,000$ lines). The calculations indicated that it is ionization from the metastable atoms which affect the charge density on the dielectric at long times. This is seen with the aid of Figure 7 in which we plot the density evolution for the ions, electrons and metastable particles in the afterglow. In Figure 7, the differences in the particle densities from the case when the data voltage is switched at $4 \mu \mathrm{s}$ are compared. The stairstep in the electron density profile is due to the acceleration technique which does not follow the true electron density once it falls below a level where it no longer affects the rest of the plasma. In the absence of these metastables, the ion and electron densities would decrease in a few microseconds Thus to reduce the decay time, it is necessary to reduce the metastable density in the afterglow. This can be done by decreasing the diffusion time of metastables (geometry changes and or neutral pressure changes) or by creating a more efficient means of metastable ionization through Penning ionization. Recent experimental results with $\mathrm{He}-\mathrm{H}_{2}$ mixtures have produced a display system with a decay time of less than $3 \mu$ s which is equivalent to over 3,000 lines addressability at $60 \mathrm{~Hz}$ frame rates[10]. This performance improvement has made the PALC display the only technology suitable for a direct view HDTV monitor with full motion and full gray-scale capability, without the use of dual scan electronics or other cost prohibitive techniques.

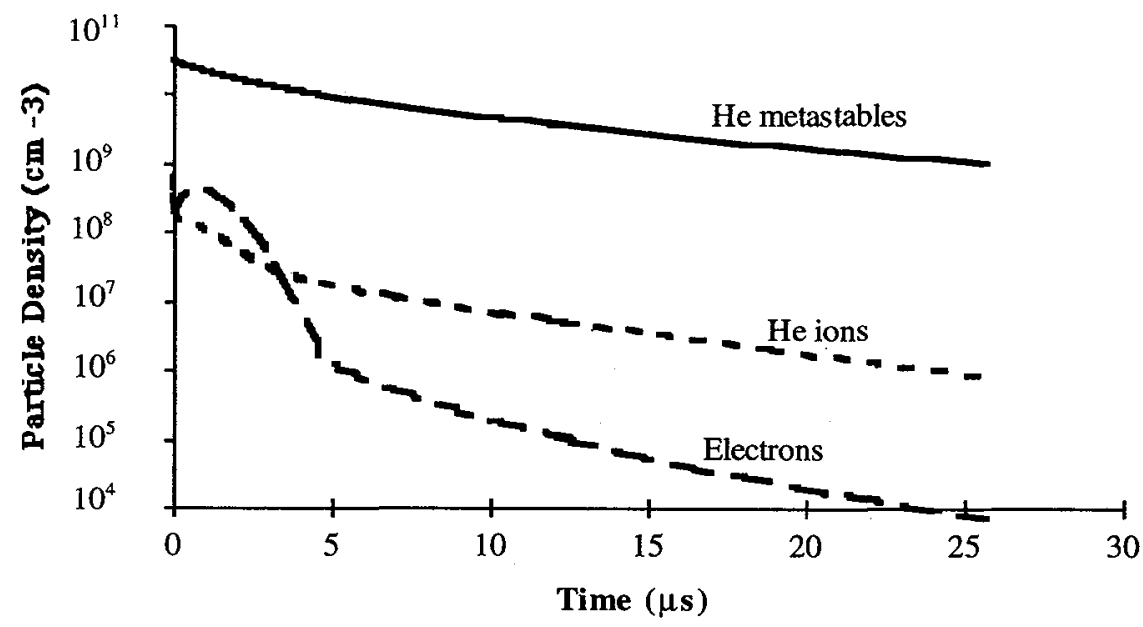

Figure 7: Density evolution of the particles in the afterglow. The symbols show the change in the density evolution when the data voltage is switched to 0 Volts at $t=4 \mu \mathrm{s}$. 


\section{SUMMARY}

The plasma addressed liquid crystal display is a new application for the properties of a glow discharge. By replacing a semiconductor switch with a three terminal plasma equivalent, a display technology that can be manufactured in large sizes was invented. Subsequent study of the properties of the plasma in these devices, a regime that has not previously seen a significant amount of attention has provided pathways to performance enhancements. The successful development of a model of the active discharge and afterglow provided insight into the underlying phenomena that were constraining the displays addressability. With a improved understanding, these limitations were removed. The result is a technology that is destined to replace the CRT in a size and format not previously thought possible.

\section{Acknowledgments}

The authors would like to acknowledge the efforts of both the process engineering and device physics groups at Technical Visions and several contributors at Lawrence Livermore National Laboratories. Several discussions with J. Moore, N. Bardsley, R. Hinchliffe, and M. Roberson have been particularly elucidating. The authors would also like to thank L. Taylor for help in the preparation of this manuscript.

\section{References}

[1] Weber L., Plasma Displays in Flat-Panel Displays and CRTs, L. Tanns Ed. (Van Nostrand Reinhold, 1985) pp. 332-407.

[2] Buzak T. "A New Active-Matrix Technique Using Plasma Addressing", Digest of Technical Papers, 1990 SID International Symposium, (Society for Information Display, 1990), pp. 420-423.

[3] Buzak T., Green P., Guthrie S., Hartley S., Hillen K., Lamer G., Martin P., Nishida D., O'Neal T., Stein W., Stinger K. and Wagner M., "A 16-inch Full-Color Plasma Addressed Active-Matrix LCD," Digest of Technical Papers, 1993 SID International Symposium, (Society for Information Display, 1993), pp. 883-886.

[4] Kakizaki T., Tanamachi S., Hayashi M., "Development of 25-in. Active-Matrix LCD Using Plasma Addressing for Video Rate High quality Displays", Digest of Technical Papers, 1995 SID International Symposium, (Society for Information Display, 1995), pp. 915-918.

[5] "Sony and Sharp Agree to Develop Jointly Big-Screen Displays" (Wall Street Journal, Sept. 20, 1996).

[6] Bubkowski J., Graves D. and Vitello P., J. Appl. Phys., in press (1996).

[7] Boeuf J. and Marode E., J. Phys. D, 15, pp. 2169 (1982).

[8] Press W., Teukolsky S., Vetterling W., Flannery B., Numerical Recipes in FORTRAN, second edition, (Cambridge University Press 1992).

[9] Kortshagen U., Parker G. and Lawler J., Phys. Rev. E, 54, pp. 6746 (1996).

[10] Ilcisin K., Buzak T., Hinchliffe R., Martin P., Roberson M., Kakizaki T., Tanamachi S., Hayashi M., Morita T., "Breakthrough Gas Mixtures for HDTV Performance in Plasma Addressed Displays," Eurodisplay '96, Proceedings of the 16th Annual International Display Research Conference, (Society for Information Display, 1996), pp. 595-599. 